Books, videos, CD-ROMs, DVDs and any other relevant items submitted for a review in the $B D J$ should be addressed to: Kate Maynard, Assistant Editor, British Dental Journal, NPG, 4-6 Crinan Street, London N1 9XW
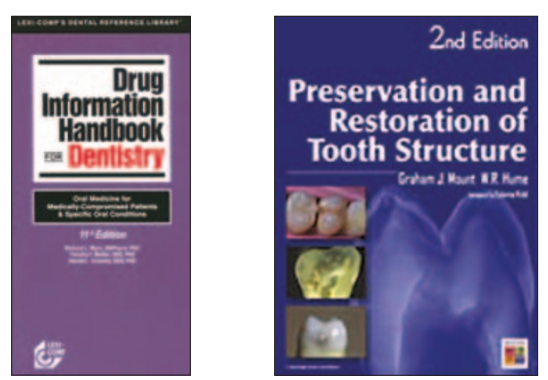

\section{Drug information handbook for dentistry}

\author{
R. L. Wynn, T. F. Meiller, H. L. Crossley \\ USA: Lexi-Comp \\ price $\$ 43.95$, pp 1898 \\ ISBN 159195102X
}

This is a concise handbook of commonly used drugs. Drugs are listed alphabetically and appropriately cross-referenced. There are six sections: the introduction, an alphabetical listing of drugs and natural products, oral medicine topics, the appendix and indexes.

The introduction describes the format giving an example of how drug and natural product information is displayed including generic/US brand names, licensed and offlicence use, drug interactions, adverse effects and pharmokinetics. Schedules outlining controlled substances and FDA pregnancy risk factor categories and advice on prescription writing are also given.

The main alphabetical drugs listing is well laid out and easy to read with very clear, concise contents, It is also crossreferenced to oral medical use with appropriate dosing schedules and adverse effects defined. Subheadings (in red) relate to local anaesthesia/vasoconstrictor precautions and effects on dental treatment.

Oral medicine topics are divided into three parts:

1. Dental management and therapeutic considerations in medically compromised patients. Groups of diseases and their drug therapies are well cross-referenced. Drug adverse reactions, interactions and possible effects on dental treatment are described. Brief, informative subsections on HIV/AIDS, STDs, viral infections and antibiotic prophylaxis are well presented. 2. Dental management and therapeutic consideration in patients with specific oral conditions and other medicine topics. It succinctly covers oral pain; antibacterial drugs and medicines used for periodontal diseases are described. There are synopses on anti-fungal and anti-viral preparations; drugs for aphthae and lichen planus, dental hypersensitivity and caries, oral sedation agents and topical prepara- tions for the management of cancer patients. Products causing and treating dry mouth are also described. Temporomandibular joint dysfunction is divorced from facial pain and has a brief list of management options.

3. Other oral medicine topics are addressed including recognising domestic violence, animal and human bite guidelines, well-defined information on chemical dependency and smoking cessation. Management of dental office emergencies gives fundamental information. A suggested reading list for all topics is given.

The appendix, pharmacological category index and alphabetical product index completes the book.

The drug section is good and constitutes the majority of the book. The much smaller section on oral medicine related conditions was rather disappointing. This book will be a useful addition to pharmacological texts but, as wisely stated at the outset, will not replace them.

\section{Yeoman}

\section{Preservation and restoration of tooth} \section{structure}

\section{G. J. Mount, W. R. Hume}

UK: Knowledge Books and Software price £99.99 (£49.99 for dental students until 30 October 2006), pp 365

\section{ISBN 192082474X}

The title of this book reinforces the most important concept of preservation of tooth tissue, which sometimes tends to be overlooked as we concentrate on techniques for restoring teeth. Emphasis is placed on minimal intervention and management of disease in the most conservative manner possible. This edition expands on changes occurring over the past 10 years. It would be useful to undergraduate and postgraduate students and also provides information for reference and updating knowledge for practitioners. As Professor Kidd says in the foreword it would be useful for 'students of all ages'!
The general layout of the book is well structured with many high quality illustrations, in particular the many excellent clinical photographs. A CD-Rom is available and images may be downloaded for teaching purposes. Material is also available on www.midentistry.org.

When exam time looms and the pressure is on, the 'be aware', 'note' and 'summary' boxes provide concise revision notes of important points. They also help to reinforce key information when reading the text.

The book runs through the structure of teeth and their supporting structures. The authors then consider disease dynamics and pulpal responses. There is an in depth review of dental caries and important consideration is given to caries risk assessment, the role of saliva and the balance between demineralisation and remineralisation. Preventive management is emphasised and the favouring of remineralisation by use of fluoride and CPP-ACP, along with reduction of the bacterial population by the use of chlorhexidine is encouraged.

After consideration of prevention, there is a techniques-based section on instruments used in cavity preparation including air abrasion, pulsed erbium lasers and chemo mechanical caries removal. Properties, including biocompatibility issues and use of dental materials are then considered. Black's cavity designs are discussed in view of current knowledge and the authors suggest a new classification based on the site and size of the lesion.

Protection of the pulp and vital pulp therapy are discussed and the difference between infected and affected dentine is highlighted. Although this tissue may be stained it is possible to remineralise this layer, rather than risk closer communication with the pulp. Atraumatic restorative treatment is discussed, along with periodontal and occlusal considerations in tooth restoration. The final chapter discusses failure of individual restorations and their management.

In summary this book covers many critical issues in restorative dentistry and is easy, interesting and enjoyable to read. 